\title{
Immobilization and Photoacoustic Spectroscopy of N-(2-Pyridyl)acetamide Cation Complexes bonded to Silica Gel
}

\author{
Claudio Airoldi, ${ }^{\star a}$ Edésio F. C. Alcântara, ${ }^{b}$ Ossamu Nakamura, ${ }^{c}$ Fernando J. da Paixão ${ }^{d}$ \\ and Helion Vargas ${ }^{d}$ \\ a Instituto de Química, Universidade Estadual de Campinas, Caixa Postal 6154, 13081-970 Campinas, \\ S.P., Brazil \\ b Instituto de Química e Geociências, Universidade Federal de Goiás, Caixa Postal 131, \\ 74690 Goiânia, Goiás, Brazil \\ 'Instituto de Física, Universidade Federal da Bahia, 40210 Salvador, Bahia, Brazil \\ ' Instituto de Física, Universidade Estadual de Campinas, Caixa Postal 6165, 13081-970 Campinas, \\ S.P., Brazil
}

\begin{abstract}
$N$-(2-Pyridyl)acetamide was covalently immobilized onto silica gel giving $1.57 \times 10^{-4} \mathrm{~mol}$ of this molecule per gram of silica. This new bidentate chelate extracts $\mathrm{MCl}_{2}(\mathrm{M}=\mathrm{Ni}, \mathrm{Co}, \mathrm{Cu})$ from ethanol and acetone solutions. The photoacoustic spectra of the solid complexed samples were obtained in the visible and near-infrared spectral regions and were analysed using ligand field theory. The calculated Racah parameters indicate a tetrahedral geometry for nickel and cobalt and a highly distorted octahedral geometry for the copper complex.
\end{abstract}

Keywords: Immobilization; Silica gel; N-(2-Pyridyl)acetamide; Photoacoustic spectroscopy; Chelating agent

In the last two decades, there has been continued interest in the investigation of chemically modified silica surfaces. The interest in these materials reflects not only fundamental questions, but also their use in a variety of applications such as chemically bonded stationary phases for chromatography, ${ }^{1,2}$ supports for catalysts ${ }^{3}$ and preconcentrating sorbents. ${ }^{4-6}$

The modification of inorganic oxides such as silica gel by coupling with functionalized organosilane agents has been widely explored. ${ }^{7}$ The main objective of this procedure is to immobilize molecules with desirable functional groups on solid surfaces. ${ }^{8}$ In many cases, the basic centres of the chemically bonded molecules act as chelating agents ${ }^{4}$ and can extract cations from aqueous ${ }^{9,10}$ or non-aqueous ${ }^{5}$ dilute solutions.

The spectroscopic characterization of metal complexes on the surface is difficult because of the low concentration, although absorption spectra can be obtained from samples prepared as discs or suspended in suitable solvents. ${ }^{5}$ Photoacoustic spectroscopy (PAS) allows direct spectra acquisition from solid samples containing cations anchored onto surfaces. This technique has been proved to be a simple and reliable method to obtain optical and thermal properties of solids samples ${ }^{1-14}$ and is especially suited to study optically opaque or highly reflecting materials such as powder or suspensions where traditional techniques are troublesome. The geometries of cation complexes immobilized on surfaces ${ }^{15}$ and solid-solution distribution measurements ${ }^{16}$ have been determined by PAS. Recently, the tetragonal distortion of copper bonded to functionalized silica has been detected. ${ }^{17}$

The aim of this publication is to report optical absorption data in the visible and near-IR spectral regions of $N-(2-$ pyridyl)acetamide divalent metal complexes immobilized on silica gel and to propose their geometry.

\section{Experimental}

\section{Materials}

All solvents were reagent grade and were purified by distillation. Silica gel (Merck) with a selected particle size of 80-170 mesh was activated by heating at $423 \mathrm{~K}$ for $7 \mathrm{~h}$ and allowed to cool in a stream of nitrogen. ${ }^{18}$ A surface area of $503 \mathrm{~m}^{2} \mathrm{~g}^{-1}$ was obtained by the BET method. Anhydrous $\mathrm{NiCl}_{2}, \mathrm{CoCl}_{2}$ and $\mathrm{CuCl}_{2}$ were obtained as described previously. ${ }^{19}$

\section{Preparations}

N-(2-Pyridyl)acetamide

This compound was prepared in $74 \%$ yield by refluxing an equimolar amount of 2-aminopyridine and acetic anhydride as described previously. ${ }^{20}$

\section{3-Chloropropylsilica Gel}

3-Chloropropyltrimethoxysilane $(0.050 \mathrm{~mol})$ dissolved in xylene $\left(5 \mathrm{~cm}^{3}\right)$ was added dropwise to a mechanically stirred suspension of activated silica gel $(25 \mathrm{~g})$ in dry xylene $\left(100 \mathrm{~cm}^{3}\right)$ and the mixture was maintained at xylene reflux temperature for $15 \mathrm{~h}$. After cooling, the solid was filtered off, washed with xylene, followed by several washings with doubly distilled water, ethanol, acetone and diethyl ether. Finally, the solid was dried in vacuo for $5 \mathrm{~h}$ at $393 \mathrm{~K}$. The degree of functionalization was monitored by digesting $c a .0 .1 \mathrm{~g}$ of this material with a large excess of pyridine and determining the chloride content by titration with standard silver nitrate solution. $^{20}$

\section{N-(2-Pyridyl)acetamide Silica Gel (Sipa)}

The preceding halogen-functionalized surface was suspended in xylene $\left(100 \mathrm{~cm}^{3}\right)$. To this mechanically stirred suspension at $373 \mathrm{~K}$, a mixture of $N$-(2-pyridyl)acetamide $(12.0 \mathrm{~g}$, $0.090 \mathrm{~mol})$ and triethylamine $(9.5 \mathrm{~g}, 0.094 \mathrm{~mol})$ dissolved in xylene $\left(30 \mathrm{~cm}^{3}\right)$ was added dropwise. This system was maintained with mechanical stirring at xylene reflux under a nitrogen atmosphere for $24 \mathrm{~h}$. After cooling, the solid was filtered off and washed as before. The resulting material was dried in vacuo for $5 \mathrm{~h}$. Functionalization was checked by determining the amount of nitrogen in the sample using Kjeldahls method. To digest the material, mercury oxide was used as catalyst to free the pyridine nitrogen, followed by treatment with sodium thiosulfate to destroy the aminomercury complexes formed. ${ }^{21}$ 


\section{Cation Extraction by Immobilized Surface}

The adsorption measurement were performed by using the batch technique in ethanol or acetone solutions. Samples of $5.0 \times 10^{-2} \mathrm{~g}$ of functionalized material were suspended with divalent metal chloride solution $\left(50 \mathrm{~cm}^{3}\right)$ in different concentrations. This suspension was mechanically stirred in a thermostat at $298.2 \pm 0.2 \mathrm{~K}$. To find the saturation of the surface, samples of immobilized material were stirred and the amount of supernatant cations was analysed by complexiometric titration with ethylenediaminetetraacetic acid (EDTA), as a function of time. For all cations in both solvents, the surface was found to be saturated within $30 \mathrm{~min}$. Therefore, $1 \mathrm{~h}$ was choosen to ensure maximum extraction. After standing for another $30 \mathrm{~min}$, the coloured solid was filtered off, washed with the solvent of the experiment and dried in vacuo for $1 \mathrm{~h}$. This same procedure with the untreated silica gel showed an absence of adsorption. ${ }^{5}$

\section{Instrumentation}

The IR spectra were obtained on a Perkin-Elmer model 180 spectrophotometer. Samples of immobilized support or divalent metal complexed surfaces were firstly pulverized. After compressing in form of discs the IR spectra were obtained by using a compensation technique. ${ }^{5}$

The photoacoustic spectra of powdered samples were obtained in the visible $(300-800 \mathrm{~nm})$ and in the near-IR $(0.8-2.8 \mu \mathrm{m})$ regions using an OAS-400 photoacoustic spectrometer. Fig. 1 illustrates the operation mode; radiation from an air-cooled high-pressure $300 \mathrm{~W}$ Xenon arc source, fitted with an integral parabolic reflection optics is focused onto a variable-speed rotating light chopper mounted at the entrance slit of a high-radiance monochromator. The monochromator has two gratings to enable the photoacoustic spectra in the UV-VIS and near-IR regions. A reflective beam-splitter passes a fraction of the dispersed radiation to a pyroelectric detector to provide source compensation and a reference signal. Source radiation is then focused onto the photoacoustic cell and sample holder assembly. The photoacoustic signal from the sample cell provides the input to the signal channel lock-in amplifier. In a similar manner the output of the pyroelectric is taken to the reference channel lock-in amplifier. The photoacoustic spectra are displayed as a chart recorder

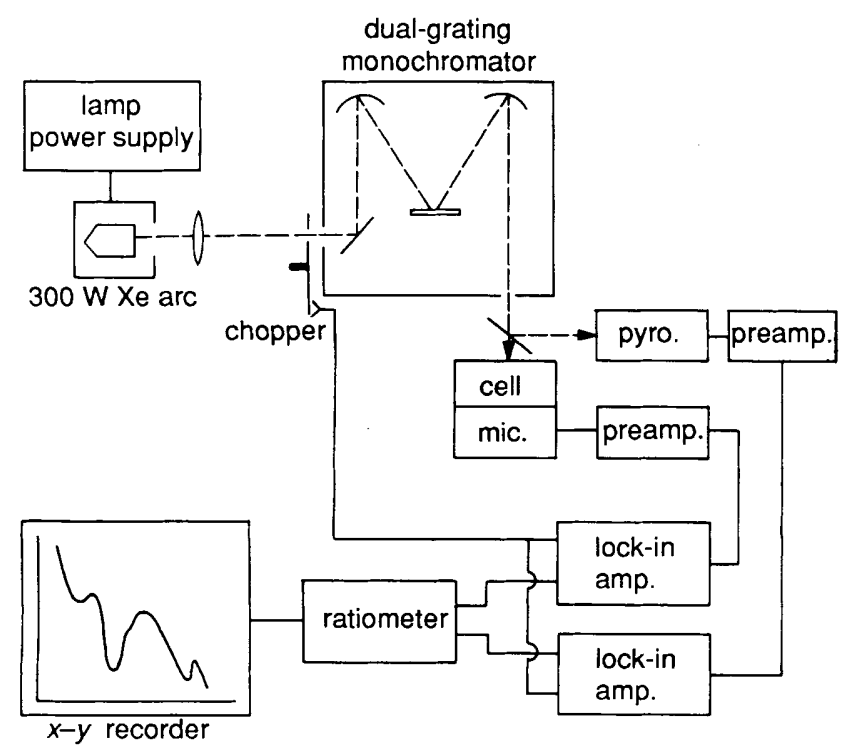

Fig. 1 Block diagram of the photoacoustic spectrometer for wavelength-dependent signal intensity as shown in Fig. 2 and 3. In this study, the experimental conditions were: chopper modulation frequency, $20 \mathrm{~Hz}$; band pass $0.032 \mathrm{~nm}$ min $^{-1}$; time constant, $1 \mathrm{~s}$; scan rate, $0.2 \mathrm{~nm} \min ^{-1}$ and sensitivity 10 and $30 \mathrm{mV},{ }^{17}$ respectively.

\section{Results and Discussion}

The preparation of a new bidentate chelate with oxygen and nitrogen basic centres attached to silica gel, is outlined in Scheme 1. Based on chlorine elemental analysis $(1.52 \%)$, $4.28 \times 10^{-4} \mathrm{~mol}$ of 3 -chloropropyl groups are bonded to each

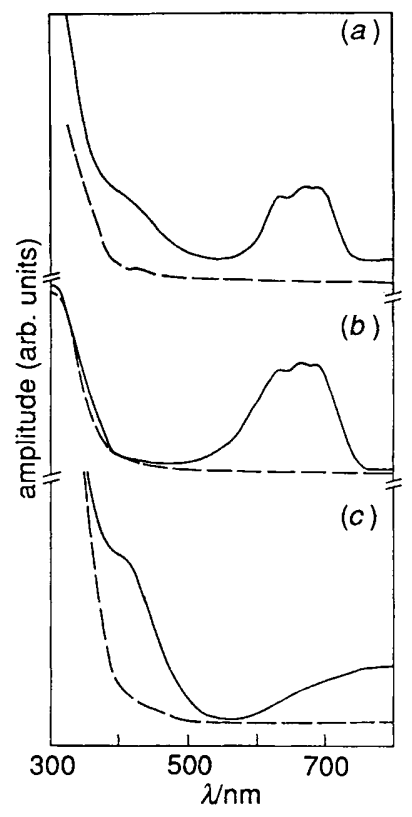

Fig. 2 Photoacoustic spectra of silica gel bonded to $N$-(2-pyridyl)acetamide. (a) Nickel, $(b)$ cobalt and $(c)$ copper in the visible region. The photoacoustic spectra of silica gel bonded to diacetamide are shown by the broken line.

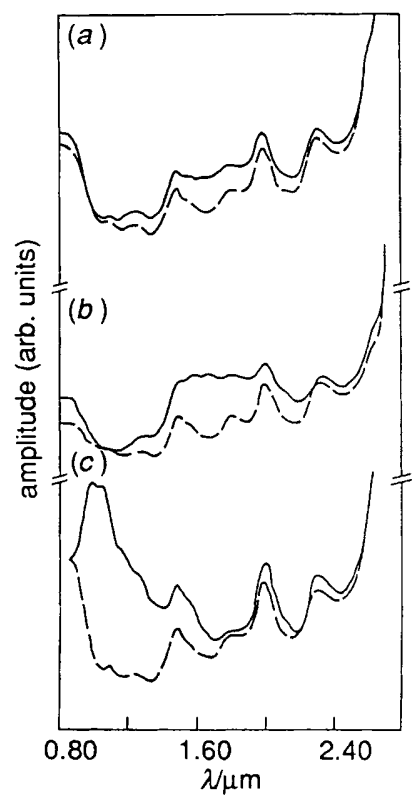

Fig. 3 Photoacoustic spectra of silica gel bonded to $N$-(2-pyridyl)acetamide. (a) Nickel, (b) cobalt, (c) copper in the near-IR region. The photoacoustic spectra of silica gel bonded to diacetamide are shown by the broken line. 


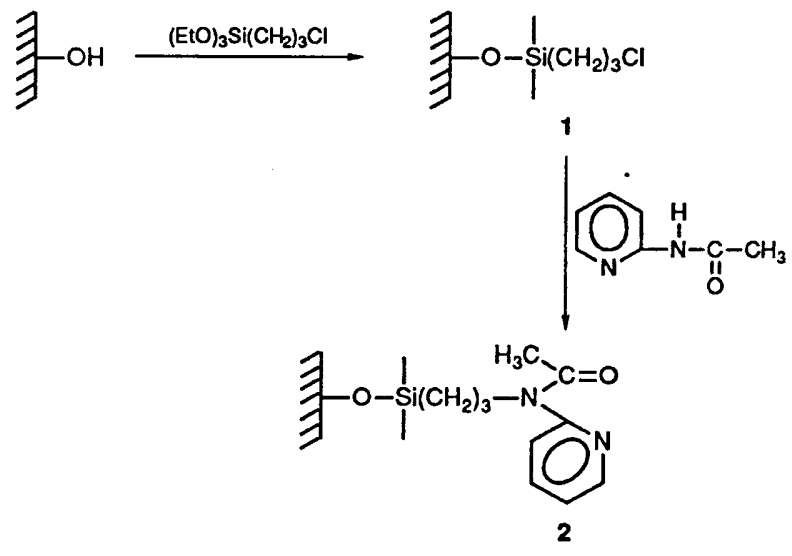

Scheme 1

gram of silica 1 . These groups are available to react with the anchoring agent in the next step. Then, the amount of nitrogen organic groups found on the final $N$-(2-pyridyl)acetamide silica gel (sipa) surface $(0.44 \%) 2$ corresponds to $1.57 \times 10^{-4} \mathrm{~mol} \mathrm{~g}^{-1}$. Since the reaction of $N$-(2-pyridyl)acetamide with the chlorosilica surface 1 has a $1: 1$ stoichiometry, these values show that about only one third of the free groups reacted. This result may reflect the steric restraints that inhibit ligand molecules from reaching the reactive centres on the surface or in adjusting to the surface pores.

The functionalized surface was pulverized and compressed into discs to obtain the IR spectra. Although matrix absortion masks the major part of the spectra of high frequencies, bands at 2980 and $1445 \mathrm{~cm}^{-1}$, which correspond to $\mathrm{CH}$ stretching and bending frequencies, respectively, were observed for surface $1 .{ }^{22}$ The stretching frequencies at 1465 and $1440 \mathrm{~cm}^{-1}$ of the $\mathrm{C}-\mathrm{C}$ and $\mathrm{C}-\mathrm{N}$ bands, respectively, appeared for the sipa surface 2 . An additional band at $1370 \mathrm{~cm}^{-1}$ is attributed to the symmetrical $\mathrm{CH}$ deformation. ${ }^{5,23,24}$ The existence of these bands supports the anchoring of $N$-(2-pyridyl)acetamide onto the silica surface.

Fig. 2 shows the photoacoustic spectra in the VIS region for a given transition metal (nickel, cobalt and copper) bonded to the sipa surface together with the spectra of the uncomplexed surface. Fig. 3 shows the corresponding photoacoustic spectra in the near-IR region, where the spectra were recorded as a function of concentration. This study was important to identify, for instance, transitions related with the $\mathrm{Ni}^{2+}$ cation. Fig. 2 and 3 show that PAS can give highquality spectra and consequently can be used as a technique to understand how the cations are bonded on immobilized surfaces.

The ligand field theory ${ }^{25}$ was applied to assign the transitions assuming that each transition-metal cation is coordinated by four or six ligands. The free ion energy levels are split by the action of ligand field and each new line observed corresponds to a transition within these energy levels.
The relative positions of the energy levels for the $3 \mathrm{~d}$ configuration in octahedral or tetrahedral symmetries can be formulated in terms of $D_{\mathrm{q}}$, which measure the ligand field strength and the Racah parameter $B$, which describe the electronic interaction energy in the $3 \mathrm{~d}$ shell. The parameters $D_{\mathrm{q}}$ and $B$, were obtained for a particular transition-metal ion bonded to sipa by fitting the observed photoacoustic bands to the possible electronic transitions given by the Tanabe and Sugano matrix. ${ }^{25}$ The fitting was made on the assumption that these transitions metals occupy octahedral (or tetrahedral) sites in the chemically modified silica surface. The parameters obtained, the assignments of the photoacoustic absorptions, and comparisons between observed and calculated band positions are given in Table 1.

Two photoacoustic absorption bands owing to divalent nickel are observed in Fig. $2(a)$ at $420 \mathrm{~nm}\left(23810 \mathrm{~cm}^{-1}\right)$ and $670 \mathrm{~nm}\left(14925 \mathrm{~cm}^{-1}\right)$ in the visible, and another in Fig. 3(a) at $1560 \mathrm{~nm}\left(6410 \mathrm{~cm}^{-1}\right)$ in the near-IR, with the ligand field parameters $D_{\mathrm{q}}=762 \mathrm{~cm}^{-1}$ and $B=1230 \mathrm{~cm}^{-1}$. The agreement between observed and calculated band positions is good assuming the nickel is in a tetrahedral site as shown in Table 1. Fig. 2(b) and $3(b)$, show, respectively, the VIS and near-IR photoacoustic spectra for divalent cobalt bonded to sipa. Two bands, one at $660 \mathrm{~nm}\left(15152 \mathrm{~cm}^{-1}\right)$ and the other at $1690 \mathrm{~nm}\left(5917 \mathrm{~cm}^{-1}\right)$ were observed. This gives $D_{\mathrm{q}}=$ $340 \mathrm{~cm}^{-1}$ and $B=724 \mathrm{~cm}^{-1}$ which are in good agreement with the observed spectra, as shown in Table 1.

The photoacoustic spectrum of divalent copper bonded to the surface of silica, shown in Fig. 2(c), is characterized by a band centred at $410 \mathrm{~nm}$ in the VIS region. Fig. 2(c) shows the spectrum of the same sample in the near-IR range. Two bands at 0.96 and $1.04 \mu \mathrm{m}$ are clearly seen. In addition, this region also presents optical bands arising from vibrational overtones of the $\mathrm{X}-\mathrm{H}$ stretch mode, where $\mathrm{X}$ is most commonly $\mathrm{C}, \mathrm{N}$, or $\mathrm{O}$. The copper(II) ion electronic configuration is $d^{9}$ and the $3 d$ levels are split to ${ }^{2} E_{g}$ and ${ }^{2} T_{2 g}$ in a ligand field of cubic symmetry. Hence, one should expect a single line in the spectrum for the ${ }^{2} E_{g} \rightarrow{ }^{2} T_{2 g}$ transition. The presence of the three lines requires an additional distortion. The ground state of this cation in an octahedral ligand field belongs to the ${ }^{2} \mathrm{E}_{\mathrm{g}}$ representation. This degenerate ground-state allows a Jahn-Teller distortion which must be considered in the interpretation of the spectrum. Thus, in a tetragonal field ${ }^{2} E_{g}$ and ${ }^{2} T_{2 g}$ split to ${ }^{2} B_{1 g}$ and ${ }^{2} A_{1 g}$ and ${ }^{2} B_{2 g}$ and ${ }^{2} E_{g}$ levels, respectively, as shown in Fig. 4. The difference in energy between ${ }^{2} \mathrm{~A}_{1 \mathrm{~g}}$ and ${ }^{2} \mathrm{~B}_{2 \mathrm{~g}}$ depends on the degree of distortion. The bands observed at 0.96 and $1.04 \mu \mathrm{m}$ should be related to the ${ }^{2} B_{1 g} \rightarrow{ }^{2} B_{2 g}$ and ${ }^{2} B_{1 g} \rightarrow{ }^{2} A_{1 g}$ transitions. The strength of the tetragonal splitting is responsible for the proximity of these levels. When the spectra with a simple hamiltonian was fitted for octahedral and tetragonal distortions, it was found that these transitions cannot be distinguishable. The band at $410 \mathrm{~nm}$ is interpreted as a ${ }^{2} \mathrm{~B}_{1 \mathrm{~g}} \rightarrow^{2} \mathrm{E}_{\mathrm{g}}$ transition due to a Jahn-Teller splitting in addition to the octahedral splitting.

The results presented here are consistent with nickel and

Table 1 Optical transitions for complexed cations immobilized on silica-gel surfaces

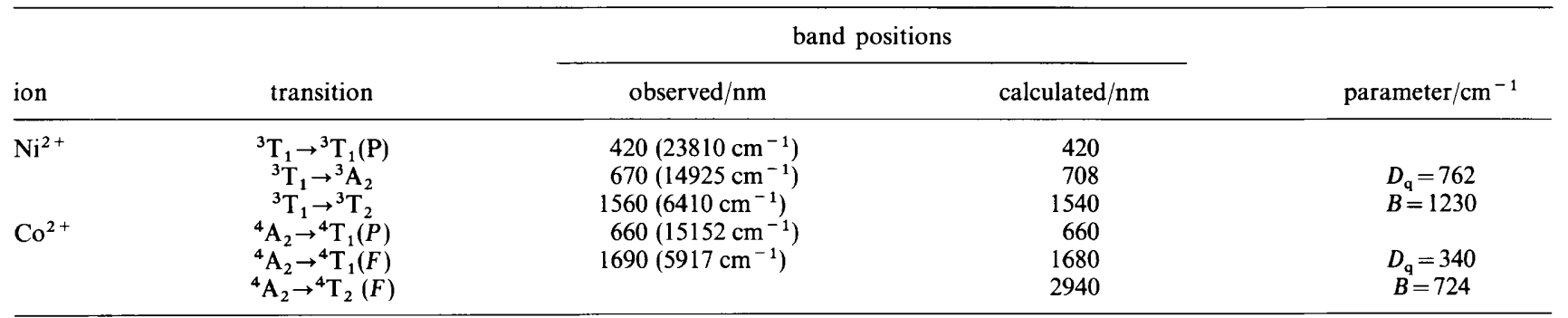




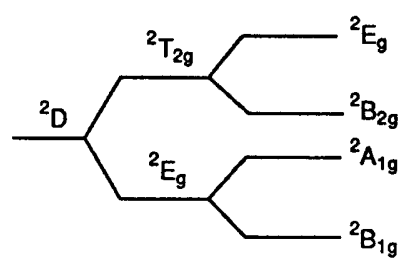

spherical octahedral tetragonal

Fig. 4 Energy diagram for copper(II) in spherical, octahedral and tetragonal symmetry fields

cobalt located in tetrahedral sites, and copper on highly distorted octahedral sites. In addition, the copper cation has a similar geometry when bonded to an immobilized oxobidentate ligand onto a silica gel surface. ${ }^{17}$

The use of PAS to characterize cation complex geometries on immobilized silica gel surfaces is rare. ${ }^{15,16}$ This study and those reported previously ${ }^{17}$ demonstrate the potential of this technique. Other investigations, which are in progress, will contribute to our understanding of the geometry of cations complexed on surfaces.

We gratefully acknowledge FAPESP and CNPq for financial support.

\section{References}

W. Wasiak, Chromatographia, 1986, 22, 147.

2 G. R. Bogart, D. E. Leyden, T. M. Wade, W. Schafer and P. W. Carr, J. Chromatogr., 1989, 483209.
3 Y. Kurusu, J. Macromol. Sci. Chem. A, 1990, 27, 1389.

4 N. Simonzadeh and A. A. Schilt, J. Coord. Chem., 1989, 20, 117

5 C. Airoldi and E. F. C. Alcântara, Colloid Surf., 1989, 39, 291.

6 M. C. Gennaro, E. Mentasti and C. Sarzanini, Polyhedron, 1986, $5,1013$.

7 U. Deschler, P. Kleinschmit and P. Panster, Angew. Chem., Int. Ed. Engl., 1986, 25, 236.

8 P. Hodge, Mendeleev Chem. J., 1989, 61, 34.

9 A. Tong, Y. Akama and S. Tanaka, Analyst, 1990, 115, 947.

10 S. Akmam, H. Ince and U. Kohlu, Anal. Sci., 1991, 7, 799.

11 H. Vargas and L. C. M. Miranda, Phys. Rep., 1988, 161, 245.

12 A. Rosencwaig, in Photoacoustics and Photoacoustic Spectroscopy, ed. P. J. Elving and J. D. Wineforder, Wiley, New York, 1980.

13 M. L. Baesso, E. C. Silva, P. P. de Abreu, F. Galembeck and H. Vargas, Phys. Chem. Glasses, 1990, 31, 122.

14 H. O. Pastore, E. Stein, E. J. Vichi, O. Nakamura, E. C. Silva and H. Vargas, J. Chem. Soc., Chem. Commun., 1990, 772.

15 R. S. Davidson, W. J. Lough, S. A. Matlin and C. L. Morrison, J. Chem. Soc., Chem. Commun., 1981, 517.

16 L. W. Burggraf, D. S. Kendall, D. E. Leyden and F. J. Pern, Anal. Chim. Acta, 1981, 129, 19.

17 E. F. C. Alcântara, C. Airoldi, O. Nakamura, H. Vargas and F. Paixão, Polyhedron, 1992, 11, 1701.

18 R. K. Iler, The Chemistry of Silica, Wiley, New York, 1979.

19 C. Airoldi and A. S. Gonç̧alves, J. Inorg. Nucl. Chem., 1978, 40, 1817.

20 A. S. Goncçalves and C. Airoldi, Polyhedron, 1989, 8, 2901.

21 C. Airoldi and A. S. Goncçalves, Colloid Surf., 1987, 28, 199.

22 L. J. Bellamy, The Infrared Spectra of Complex Molecules, Menthuen, London, 1966.

23 C. K. Jörgensen, in Absorption Spectra and Chemical Binding in Complexes, Pergamon Press, Oxford, 1962

24 C. Airoldi, F. S. Dias, J. G. Espinola and G. F. de Sá, J. Chem. Res. (S), $1980,142$.

25 Y. Tanabe and S. Sugano, J. Phys. Soc. Jpn., 1958, 13, 880.

Paper 2/05887A; Received 3rd November, 1992 